\title{
Quantum mechanical rotation of a photon polarization by Earth's gravitational field
}

\author{
Hansol Noh ${ }^{1,2}$, Paul M. Alsing ${ }^{3 凶}$, Doyeol Ahn $\mathbb{1}^{1,4,5 凶}$, Warner A. Miller (iD) and Namkyoo Park (iD ${ }^{2}$
}

We describe the quantum mechanical rotation of a photon state, the Wigner rotation-a quantum effect that couples a transformation of a reference frame to a particle's spin, to investigate geometric phases induced by Earth's gravitational field for observers in various orbits. We find a potentially measurable quantum phase of the Wigner rotation angle in addition to the rotation of standard fame, the latter of which is computed and agrees well with the geodetic rotation. When an observer is in either a circular or a spiraling orbit containing non-zero angular momentum, the additional quantum phase contributes $10^{-6}$ degree to $10^{-4}$ degree respectively, depending on the altitude of the Earth orbit. In the former case, the additional quantum phase is dominant over the near-zero classical geodetic rotation. Our results show that the Wigner rotation represents a non-trivial semiclassical effect of quantum field theory on a background classical gravitational field.

npj Quantum Information (2021)7:163; https://doi.org/10.1038/s41534-021-00471-6

\section{INTRODUCTION}

As threats for global secure key distribution have increased with the exponential growth of computing powers, free-space-based quantum key distribution (QKD) systems have been actively pursued with the recent deployment of the Quantum Experiments at Space Scale network and Micius satellite ${ }^{1-20}$. While several proposals for quantum communication between a LEO satellite and an optical ground station have been suggested, such as SPACEQEST and QEYSSAt projects, an experiment in the regime where a quantum system evolves in curved space-time has never been fully assessed; most of them have mainly considered quantum optics rather than general relativistic phenomena of quantum states at large scales ${ }^{6-9}$. The gravitational field adds a measurable contribution to the quantum bit error rate (QBER) along the world line of the quantum state as the altitude changes $^{9}$. The gravitational field also causes the red shift ${ }^{21,22}$ of the photon, which should be compensated for the QKD system. Therefore, it is important in these environments to understand the interplay of quantum theory and gravitation as the photon state, propagating between the ground station and the satellite, carries the quantum information. It was found that the polarization encoding is a reasonable option for a microsatellite-based quantum-limited communication in an LEO-to-ground link due to its stable propagation through the atmosphere, whereas timebin encoding is widely used in the fiber networks ${ }^{23}$. A big challenge in this kind of system is polarization reference-frame synchronization between the LEO satellite and the optical ground station to implement the QKD protocol reliably in the gravitational field ${ }^{23}$ which induces the Lense-Thirring (frame-dragging) and de Sitter (geodetic precession) effects ${ }^{24-27}$. Furthermore, an experiment has been proposed to measure these gravitation-induced effects on quantum states near Earth using a ferromagnetic gyroscope whose angular momentum is dominated by atomic electron spins ${ }^{28}$. Nevertheless, while there have been works on the change of polarization and linear momentum of a photon as it propagates through a gravitational field in a general relativistic point of view ${ }^{29-32}$, the investigation of quantum systems with the general relativity has been mainly focused on the proper time and time dilation effect ${ }^{33,34}$.

Describing photon states observed by a moving observer (e.g., a satellite) in curved spacetime requires the understanding of both quantum mechanics and general relativity, two essential branches of modern physics. One of the conceptual barriers for the relativistic treatment of quantum information is the difference in the role played by the wave fields and the state vectors in relativistic quantum theory. In non-relativistic quantum mechanics, the wave function of Schrödinger's equation gives the probability amplitude that can be used to define conserved particle densities or density matrices. However, it was discovered that relativistic equations are only indirect representations for probability waves of a single particle ${ }^{35}$. In 1939, Wigner proposed the idea that the quantum states of relativistic particles can be formulated without the use of wave equations ${ }^{36}$. He showed that the states of a free particle are given by a unitary irreducible representation of the Poincaré group. In Wigner's formulation, relativistic-particle states in different inertial frames are related by a little group element in the irreducible representation of the Poincaré group, called Wigner rotation ${ }^{35-43}$.

While Wigner's original proposal was for special relativity, there have been several attempts to extend it to the domain of general relativity ${ }^{39,41-43}$. It has been shown that moving-particle states in curved spacetime are transformed into each other by Wigner rotation ${ }^{39,41-43}$ by introducing tetrads (frame fields) to define local coordinates $^{44}$ since extending Wigner's group to curved spacetime requires the standard local laboratory at every event ${ }^{29}$. For free-space QKD systems, the gravitational field induces a rotation of the linear polarization of a photon observed between an earth ground station and a satellite in the near-Earth orbit. Thus, it would be a particularly important problem, from not only a fundamental point of view for testing general relativistic effects on quantum theory but also from an application point of view for

${ }^{1}$ Department of Electrical and Computer Engineering and Center for Quantum Information Processing, University of Seoul, 163 Seoulsiripdae-ro, Dongdaemun-gu, Seoul 02504, Republic of Korea. ${ }^{2}$ Department of Electrical and Computer Engineering, Seoul National University, Seoul 08826, Republic of Korea. ${ }^{3}$ Air Force Research Laboratory, Information Directorate, Rome, NY 13441, USA. ${ }^{4}$ Physics Department, Charles E. Schmidt College of Science, Florida Atlantic University, 777 Glades Road, Boca Raton, FL 33431-0991, USA. ${ }^{5}$ Peta Lux Inc., 3F TLi Building, 12 Yanghyeon-ro, 405 beon-gil, Jungwon-gu, Seongnam-si, Gyeonggi-do 13438, Republic of Korea. ${ }^{凶}$ email: paul.alsing@us.af.mil; dahn@uos.ac.kr 
precision quantum metrology and free-space quantum communication, to be able to measure this Wigner rotation angle (WRA).

In this paper, we demonstrate the existence of a non-trivial Wigner rotation experienced by photons sent non-radially from Earth ground station to a free-falling observer with non-zero angular momentum and various altitudes. To focus on the existence of a measurable WRA, we simplify the spacetime of Earth, considering only the monopole of Earth. Examining two orbits, circular and spiral, both with non-zero quantum-phase Wigner rotation components, it is found that there is a potentially measurable quantum phase of the WRA in addition to the rotation of standard fame, which agrees well with the geodetic rotation, measured by Gravity Probe B in $2011^{24-26}$, and in the former case, the additional quantum phase is dominant to the near-zero geodetic rotation. Our results show that the Wigner rotation involves a non-trivial semi-classical effect of quantum field theory on a background classical gravitational field in addition to classical geodetic precession. This finding could open up the testbed to probe gravitational effects on various quantum phenomena in a satellite by the interplay of two pillars of physics.

\section{RESULTS}

\section{Irreducible representation of the Wigner rotation}

Considering only the monopole of Earth, we model the spacetime around Earth with Schwarzschild spacetime where tetrad fields can be globally defined as orientation-preserved coordinate bases ${ }^{40,45,46}$, and the $(-+++)$ metric signature is used. Furthermore, it is also assumed ${ }^{45}$ that quantum field theories on spacetime admit a spinor structure ${ }^{37,44,47}$ which will be employed for the quantum state of the photon with a given polarization.

While there has been much renewed interest in the effects of the gravitational field on quantum systems, especially in relation to the effect of accelerated motion and horizons on quantum entanglement ${ }^{48-51}$ inspired by the seminal work of Hawking ${ }^{52}$ and Unruh $^{53}$, less work has been performed on an experimental assessment of the regime in which quantum systems evolve on classical curved spacetime. Our model could provide a testbed for probing the gravitational effects on quantum systems.

The Hilbert space vector of a photon is defined in a local inertial frame spanned by a tetrad, $e_{\hat{a}}^{\mu}(\mathbf{x}), \hat{a}$ and $\mu=0,1,2,3$, which satisfies $g_{\mu v}(\mathbf{x})=\eta_{\hat{a} \hat{b}} e_{\mu}^{\hat{a}}(\mathbf{x}) e_{v}{ }^{\hat{b}}(\mathbf{x})$ and transforms in a way that $\bar{e}_{\hat{a}}^{\mu}(\overline{\mathbf{x}})=\frac{\partial \bar{X}^{\mu}}{\partial x^{\nu}} e_{\hat{a}}^{v}(\mathbf{x})$ and $\bar{e}_{\hat{a}}^{\mu}(\mathbf{x})=\Lambda_{\hat{a}}^{\hat{b}} e_{\hat{b}}^{\mu}(\mathbf{x})$ under general coordinate and local Lorentz transformations, respectively. Throughout the paper, we use hatted Latin letters for local inertial coordinates and Greek letters for general coordinates. The tetrad $e_{\hat{a}}^{\mu}(\mathbf{x})$ carries two indices: (i) a 'world index' $\mu$ that transforms under general coordinate transformations of general relativity, and (ii) a local tangent plane index $\hat{a}$ which transforms under local, $\mathbf{x}$-dependent Lorentz transformation $\Lambda(\mathbf{x})$. Quantities such as $p_{\hat{a}}(\mathbf{x})=$ $e_{\hat{a}}^{\mu}(\mathbf{x}) p_{\mu}(\mathbf{x})$ are the projection of the general relativistic world momentum vector $p_{\mu}(\mathbf{x})$ onto the axes (three spatial, one temporal) of the observer's local laboratory frame ${ }^{54}$ where the metric is locally flat such that special relativity holds over distances over which the curvature can be considered as essentially constant. The tetrad, therefore, explicitly embodies Einstein's Equivalence Principle through the index $\hat{a}$. The local Lorentz transformation $\Lambda_{\hat{a}}^{\hat{b}}(\mathbf{x})$ transforms between different instantaneous states of motion of the observer (e.g., stationary, freely falling, circular motion, or arbitrary motion) within the same Lorentz tangent plane at the position $\mathbf{x}$, i.e., the tetrad is constructed to define (and describes) the observer's instantaneous state of motion at each point $\mathbf{x}$.

A variation of a tetrad under an infinitesimal translation from $\mathbf{x}$ to $\mathbf{x}+\delta \mathbf{x}$ is described by parallel transport to compare two vectors in the same tangent plane without a change of the vectors such that ${ }^{40,46}$

$\delta\left(e_{\hat{a}}{ }^{\mu}\right)=\bar{e}_{\hat{a}}{ }^{\mu}(\mathbf{x}+\delta \mathbf{x})-\bar{e}_{\hat{a}}{ }^{\mu}(x) \rightarrow \delta x^{\lambda} \nabla_{\lambda} e_{\hat{a}}{ }^{\mu}(\mathbf{x})$.

For the case that wave vector of a photon is measured in the observer's laboratory, local covariant components of the wave vector, $k_{\hat{a}}(\mathbf{x})=e_{\hat{a}}^{\mu}(\mathbf{x}) k_{\mu}(\mathbf{x})$, are changed along the photon's geodesic from $x^{\mu}$ to $x^{\mu}+k^{\mu}(\mathbf{x}) \delta \xi$ such that

$\delta k_{\hat{a}}(\mathbf{x})=\delta\left(e_{\hat{a}}^{\mu}(\mathbf{x})\right) k_{\mu}(\mathbf{x})+e_{\hat{a}}{ }^{\mu}(\mathbf{x}) \delta k_{\mu}(\mathbf{x}) ; \delta k_{\mu}(\mathbf{x})=\mathrm{d} \xi \nabla_{\mathbf{k}} k_{\mu}(\mathbf{x})$.

Since a photon state in curved spacetime follows a null geodesic in the geometric optics limit $^{55}$ and under a local infinitesimal change of a tetrad (which is antisymmetric ${ }^{39-41}$ ), Eq. (2) can be rewritten as

$$
\begin{aligned}
k_{\hat{a}}(\mathbf{x}) \rightarrow k_{\hat{a}}^{\prime}(\mathbf{x}) & \equiv k_{\hat{a}}(\mathbf{x})+\delta k_{\hat{a}}(\mathbf{x}) \\
& =\left(\delta_{\hat{a}}^{\hat{b}}+\lambda_{\hat{a}}^{\hat{b}}(\mathbf{x}) \mathrm{d} \xi\right) k_{\hat{b}}(\mathbf{x})=\Lambda_{\hat{a}}^{\hat{b}}(\mathbf{x}) k_{\hat{b}}(\mathbf{x}),
\end{aligned}
$$

where $\lambda_{\hat{a}}^{\hat{b}}(\mathbf{x})=\left(\nabla_{\mathbf{k}} e_{a}^{v}(\mathbf{x})\right) e_{v}^{\hat{b}}(\mathbf{x})$. In other words, the effect of an infinitesimal translation can be considered as an infinitesimal local Lorentz transformation given by $\Lambda_{\hat{a}}{ }^{b}(\mathbf{x})=\delta_{\hat{a}}{ }^{b}+\lambda_{\hat{a}}{ }^{b}(\mathbf{x})^{39-43}$.

A Lorentz transformation, $\Lambda$, has the one-dimensional representations for a photon state with the helicity, $\sigma$, given by ${ }^{37}$

$U(\Lambda)|\mathbf{k}, \sigma\rangle=\sum_{\sigma^{\prime}} D_{\sigma / \sigma}(W(\Lambda, \mathbf{k}))\left|\Lambda \mathbf{k}, \sigma^{\prime}\right\rangle$.

$W(\Lambda, \mathbf{k})$ is the Wigner's little group element, defined as $W(\Lambda, \mathbf{k})=$ $L^{-1}(\Lambda \mathbf{k}) \Lambda L(\mathbf{k})$ and $D(W)$ is the irreducible representation of $W . L(\mathbf{k})$ is the Lorentz transformation such that $L(\mathbf{p}) \mathbf{k}=\mathbf{p}$. Accordingly, a displacement of a photon state leads to a residual quantum phase called the WRA. To get an explicit expression of the irreducible unitary representation of a Lorentz transformation, we use the canonical group homomorphism between the proper Lorentz group and its double cover, $\operatorname{SL}(2, \mathbb{C})$; a wave vector $\mathbf{k}$ of a photon is mapped to a Hermitian matrix $K$ via $K=\sigma_{\hat{a}} k^{\hat{a}}$, where $\sigma_{\hat{a}}, \hat{a}=1,2$, and 3 , are the Pauli matrices, and $\sigma_{\hat{0}}$ is the $2 \times 2$ identity matrix. A Lorentz transformation is represented by the similarity transformation such that

$A K A^{\dagger}=\Lambda^{\mu}{ }_{v} k^{v} \sigma_{\mu}$

with an element $A=\left(\begin{array}{ll}a & \beta \\ \gamma & \delta\end{array}\right)$ of $\operatorname{SL}(2, \mathbb{C})$. For an infinitesimal homogeneous Lorentz, the matrix A can be expanded in terms of $d \xi$ such that

$A=\left(\begin{array}{cc}a & \beta \\ \gamma & \delta\end{array}\right)=I+\tilde{A} \mathrm{~d} \xi=I+\left(\begin{array}{cc}\tilde{a} & \tilde{\beta} \\ \tilde{\gamma} & -\tilde{a}\end{array}\right) \mathrm{d} \xi$.

Substituting Eq. (6) into Eq. (5), multiplying $\sigma_{\hat{a}}$ both sides, and then taking a trace on both sides, we can get the following equations

$\lambda_{\hat{b}}^{\hat{a}}=\frac{1}{2} \delta^{\hat{a} \hat{c}} \operatorname{tr}\left(\sigma_{\hat{b}} \sigma_{\hat{c}} \tilde{A}+\sigma_{\hat{c}} \sigma_{\hat{b}} \tilde{A}^{\dagger}\right)$,

where $\operatorname{tr}(A)$ is the trace of $A$. That is, we obtain $\tilde{a}, \tilde{\beta}$, and $\tilde{\gamma}$ in terms of $\lambda_{\hat{b}}^{\hat{a}}$ such that

$$
\begin{aligned}
& \tilde{a}=\frac{1}{2}\left(\lambda_{\hat{}^{\hat{0}}}+i \lambda^{\hat{1}}{ }_{\hat{2}}\right) \\
& \tilde{\beta}=\frac{1}{2}\left[\left(\lambda^{\hat{0}}{ }_{\hat{1}}+\lambda^{\hat{3}}{ }_{\hat{1}}\right)+i\left(-\lambda^{\hat{0}}{ }_{\hat{2}}+\lambda^{\hat{2}}{ }_{\hat{3}}\right)\right] \\
& \tilde{\gamma}=\frac{1}{2}\left[\left(\lambda^{\hat{0}_{\hat{1}}}-\lambda^{\hat{3}}{ }_{\hat{1}}\right)+i\left(\lambda_{\hat{2}}^{\hat{0}_{\hat{2}}}+\lambda^{\hat{2}}{ }_{\hat{3}}\right)\right] .
\end{aligned}
$$

The corresponding irreducible unitary representation of the little group element for a massless particle is ${ }^{43,56}$

$$
\boldsymbol{e}^{i(\psi(\Lambda, k) / 2)}=\frac{\left[a\left(1+n^{\hat{3}}\right)+\beta n_{+}\right] b+\left[\gamma\left(1+n^{\hat{3}}\right)+\delta n_{+}\right] c^{*}}{a \sqrt{b\left(1+n^{\hat{3}}\right)}},
$$



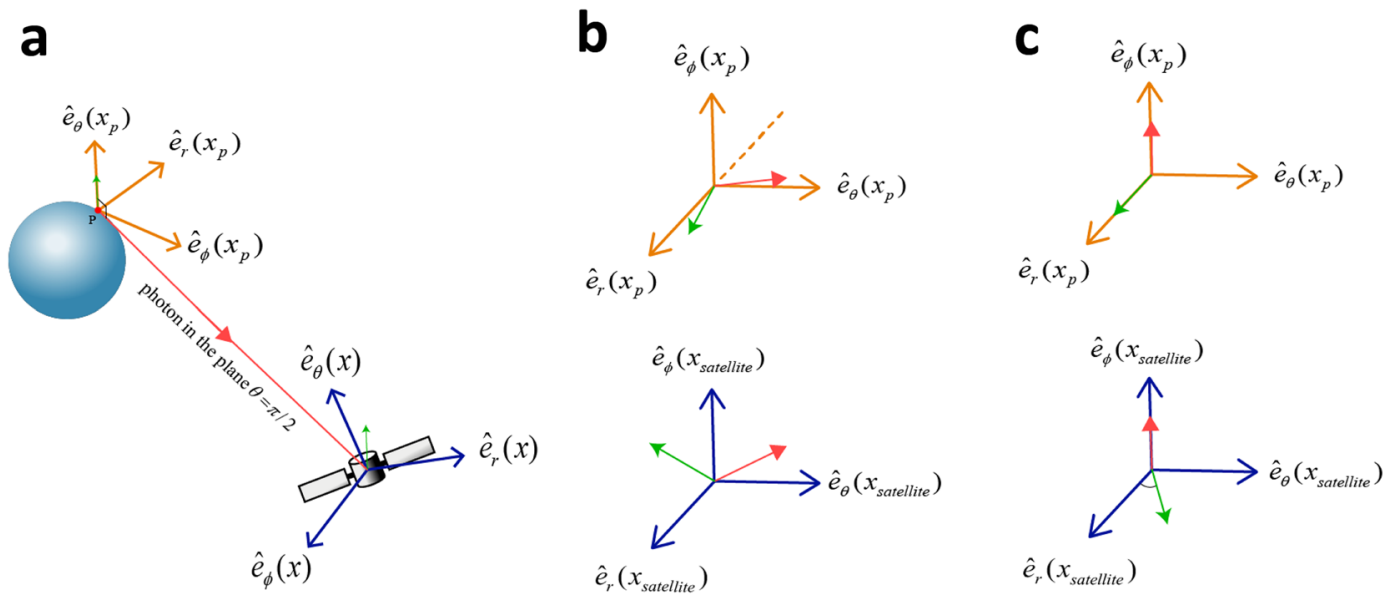

Fig. 1 Schematics of the Earth-satellite system. The Earth-Satellite system and corresponding coordinates. a A photon's geodesic and its polarizations are represented by a red line and light-green arrows, respectively. b, $\mathbf{c}$ The wave vector and polarization of the photon are measured in each local frame (b) and compared in the standard frame (c).

where $\psi(\Lambda, \mathbf{k})$ is the WRA. Detailed expressions for $a, b, c$, and $d$ are given in the Supplementary Information (SI). Thus, a local infinitesimal Lorentz transformation $\Lambda(\mathbf{x})$ leads to an infinitesimal Wigner rotation angle (IWRA) $\tilde{\psi}$, and the total WRA $\psi$ can be formally obtained by a time-ordered integration of IWRs over the geodesic trajectory $\mathbf{x}(\xi)$ of the photon such that

$$
\boldsymbol{e}^{\boldsymbol{i} \psi(\Lambda, \vec{n})}=T \exp \left[\boldsymbol{i} \int \tilde{\psi}\left(\Lambda(\mathbf{x}(\xi)), n^{\hat{i}}(\xi)\right) \mathrm{d} \xi\right]
$$

where $n^{\hat{i}}=k^{\hat{i}}(\mathbf{x}) / k^{\hat{0}}(\mathbf{x})$, and $T$ is the time-ordering operator. While the Wigner little group has finite irreducible unitary representation in the standard frame as shown in Eq. (9), a boost has no finite irreducible representation in general ${ }^{37,57,58}$. Thus, the Wigner little group has been introduced to make finite irreducible unitary representations of arbitrary Lorentz transformations for a photon's polarization vector as a gauge-fixing of photon's 4-vector potential $\left.\right|^{3743,57-59}$. For a polarization vector of photons described by $a^{\hat{a}}(\mathbf{x})=\frac{1}{(2 \pi)^{3 / 2}} \int \frac{d^{3}(h k)}{\sqrt{2 h k^{0}}} \sum_{\sigma=1,-1}\left[a(\hat{\mathbf{k}}, \sigma) \varepsilon_{\sigma}^{\hat{a}}(\hat{\mathbf{k}}, \sigma) \boldsymbol{e}^{i \mathbf{k} \cdot \mathbf{x}}+a^{\dagger}(\hat{\mathbf{k}}, \sigma) \varepsilon_{\sigma}^{\hat{a}}(\hat{\mathbf{k}}, \sigma)^{*} \boldsymbol{e}^{-i \mathbf{k} \cdot \mathbf{x}}\right]$, the helicity vectors, $\varepsilon_{\sigma}^{\mu}(\hat{\mathbf{k}}, \sigma)$, has the relation under a Lorentz transformation $\Lambda$ such that ${ }^{37}$

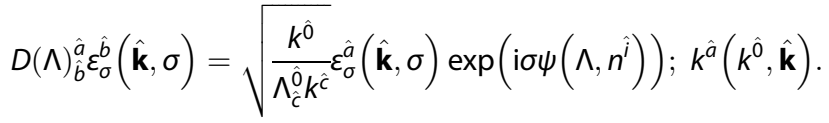

Here, $a(\hat{\mathbf{k}}, \sigma)$ and $a^{\dagger}(\hat{\mathbf{k}}, \sigma)$ are annihilation and creation operators, respectively. Correspondingly, the polarization vector of a photon, $\varepsilon_{\varphi}^{\hat{a}}$ is transformed in the standard frame such that ${ }^{43}$

$$
\begin{aligned}
D(\Lambda) \varepsilon_{\phi}^{\hat{a}} & =\left(e^{i \phi} \varepsilon_{\sigma=1}^{\hat{a}}+e^{-i \phi} \varepsilon_{\sigma=-1}^{\hat{a}}\right) \\
& =\left(e^{i\left(\phi+\psi\left(\Lambda, n^{\hat{i}}\right)\right)} \varepsilon_{\sigma=1}^{\hat{a}}+e^{-i\left(\phi+\psi\left(\Lambda, n^{\hat{i}}\right)\right)} \varepsilon_{\sigma=-1}^{\hat{a}}\right) \\
& =R_{\hat{z}}\left(\psi\left(\Lambda, n^{\hat{i}}\right)\right) \varepsilon_{\phi}^{\hat{a}}=\varepsilon_{\phi^{\prime}}^{\hat{a}},
\end{aligned}
$$

where $\varphi \prime=\varphi+\psi\left(\Lambda, n^{\hat{i}}\right)$ and $R_{\hat{z}}(\psi)$ is the rotation about $\hat{z}$-axis by the total WRA.

\section{Earth-satellite system}

We consider an Earth-satellite system depicted in Fig. 1. Figure 1a shows the Earth-Satellite system and corresponding coordinates. A photon is sent along its geodesic, represented by the red line, and its polarization, represented by the light-green arrows, is measured in the local frame of a satellite. The Schwarzschild metric is used to model spacetime around Earth and choose spacelike components of the tetrads so that the first, second, and third axis of the local frames become unit vectors of Schwarzschild coordinates $r, \theta$, and $\varphi$ at infinity, i.e., $\hat{e}_{\hat{a}}{ }^{\mu}\left(\mathbf{x}_{\infty}\right) \equiv \hat{e}_{b}{ }^{\mu}$ where $\hat{a}=1,2$, and 3 correspond to $b=r, \theta$, and $\varphi$, respectively. To compare the polarization measured at the surface of Earth and the satellite, the standard frame is introduced in which a wave vector of the photon is aligned to the third axis of the observer's local frames (Fig. 1b, c).

We consider the following four observer trajectories: a stationary observer, a radially free-falling observer, a freefalling observer with non-zero angular momentum in a circular, and a spiraling orbit ${ }^{54}$ (Fig. 2a). To define non-spinning local frames, we apply Fermi-walker transport and parallel transport conditions for the stationary and free-falling observers respectively. Detailed works are given in the Supplementary Information. We note that the definition of polar coordinates induces a non-physical rotation in local frames, which must be canceled out. In other words, if the photon's geodesic is in the equatorial plane $\theta=\pi / 2$ (Fig. 2b, c), the unit vector of the coordinate $r$ is rotated as the coordinate $\varphi$ changes. Thus, the observer is assumed to move in the plane $\hat{e}_{r}-\hat{e}_{\theta}$, i.e., the constant- $\varphi$ plane (Fig. $2 \mathrm{~b}, \mathrm{c}$ ), and $\varphi$-axis is chosen as the third axis of the local frames to cancel out the polar-coordinate-induced rotation when a wave vector is aligned to $\varphi$-axis for polarization comparison. It is worth mentioning that the Wigner rotation has a zero angle in special relativity if the direction of boost (observer) and the wave vector (photon) both lie in the $\hat{x}-\hat{z}$ plane or the $\hat{y}-\hat{z}$ plane. However, if a photon moves in the $\hat{x}-\hat{y}$ plane and an observer in the orthogonal $\hat{x}-\hat{z}$ plane, the WRA is not necessarily zero ${ }^{43,56}$. Correspondingly, by the equivalence principle, since our observers are assumed not to move in the plane $\hat{e}_{\hat{1}}{ }^{\mu}(\mathbf{x})-\hat{e}_{\hat{3}}{ }^{\mu}(\mathbf{x})$, and the photon's geodesic remains in the equatorial plane $\theta=\pi / 2$ (Fig. 2b, c), the WRA will again be non-zero.

Timelike components of the corresponding tetrads, $\hat{e}_{t}{ }^{\mu}$, are set to the 4-velocity vector of a massive particle (e.g., satellite), moving along a geodesic corresponding to each trajectory, describing the local frame of the observer. The 4-velocity vectors of the observers and the wave vector of the photon are obtained in terms of conserved quantities defined from Killing vectors of Schwarzschild spacetime (see Supplementary Eq. (5)). 
a

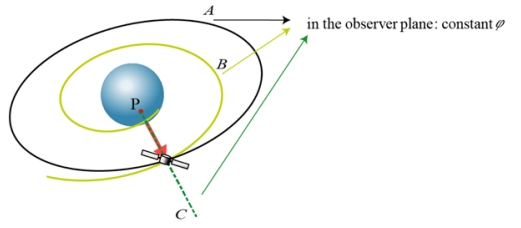

b

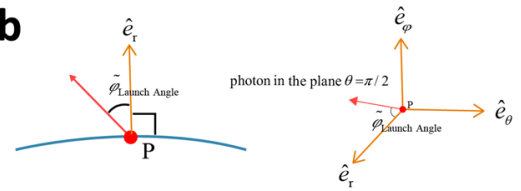

C

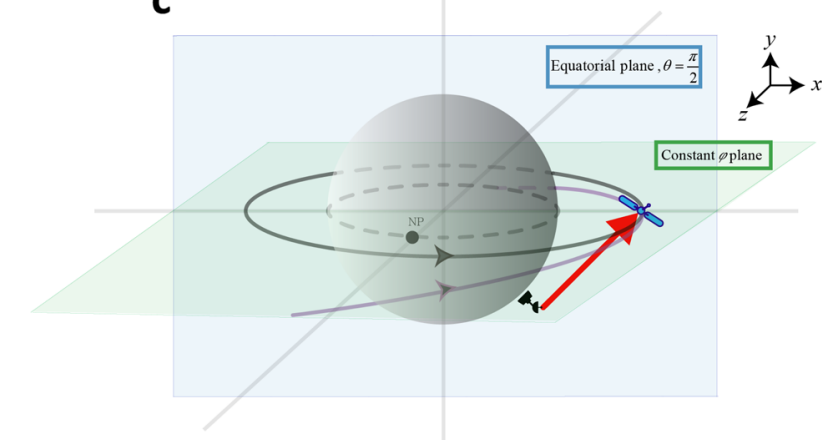

Fig. 2 Schematics of the trajectory of the observer (satellite) and photon. The geodesics of a photon traveling lies in the equatorial plane, $\theta=\pi / 2$ and the observer's geodesics is lying in the constant $\varphi$-plane (the point NP on the sphere represents the North Pole). a A, $B$, and $C$ represent the geodesics of massive free-falling observers radially, in a circular orbit, and spiraling orbit, respectively. b, c The non-radial launching angle of a photon from the surface of the Earth to the satellite in a Fig. 2c circular (gray) and spiraling (purple) orbit in the constant $-\varphi$ plane.

For circular orbits, by applying the conditions of orthogonality and non-spinning frame, tetrads have the form

$$
\begin{aligned}
\left(e_{\hat{0}}\right)^{\mu}(\mathbf{x})= & \left(e_{\mathrm{t}}\right)^{\mu}(\mathbf{x})=\left(\frac{1}{\sqrt{1-\frac{3 r_{s}}{2 r}}}, 0 \frac{1}{r} \sqrt{\frac{r_{s}}{2 r}} \frac{1}{\sqrt{1-\frac{3 r_{s}}{2 r}}}, 0\right) \\
\left(e_{\hat{1}}\right)^{\mu}(\mathbf{x})= & \left(e_{\mathrm{r}}\right)^{\mu}(\mathbf{x})\left(-\sqrt{\frac{r_{s}}{2 r}} \frac{\sin \tilde{\Theta}(r)}{\sqrt{1-\frac{3 r_{s}}{2 r}} \sqrt{1-\frac{r_{s}}{r}}}, \sqrt{1-\frac{r_{s}}{r}} \cos \tilde{\Theta}(r),\right. \\
& \left.-\frac{1}{r} \frac{\sqrt{1-\frac{r_{s}}{r}} \sin \tilde{\Theta}(r)}{\sqrt{1-\frac{3 r_{s}}{2 r}}}, 0\right) \\
\left(e_{\hat{2}}\right)^{\mu}(\mathbf{x})= & \left(e_{\theta}\right)^{\mu}(\mathbf{x})\left(\sqrt{\frac{r_{s}}{2 r}} \frac{\cos \tilde{\Theta}(r)}{\sqrt{1-\frac{3 r_{s}}{2 r}} \sqrt{1-\frac{r_{s}}{r}}}, \sqrt{1-\frac{r_{s}}{r}} \sin \tilde{\Theta}(r),\right. \\
& \left.\sqrt{1-\frac{r_{s}}{r}}, \frac{1}{r} \frac{\sqrt{1-\frac{r_{s}}{r}} \cos \tilde{\Theta}(r)}{\sqrt{1-\frac{3 r_{s}}{2 r}}}, 0\right) \\
\left(e_{\hat{\jmath}}\right)^{\mu}(\mathbf{x})= & \left(e_{\varphi}\right)^{\mu}(\mathbf{x})=(0,0,0, \csc \theta / r),
\end{aligned}
$$

where $\tilde{\Theta}(r)=\sqrt{1-\frac{3 r_{s}}{2 r}}\left(\theta-\theta_{0}\right)$. Likewise, for spiraling orbits, the tetrad can be written such that

$$
\begin{aligned}
& \left(e_{\hat{0}}\right)^{\mu}(\mathbf{x})=\left(e_{\mathrm{t}}\right)^{\mu}(\mathbf{x})=\left(\frac{1}{\left(1-\frac{r_{s}}{r}\right)},-\sqrt{\frac{r_{s}}{r}} \cos \Theta(r),-\frac{1}{r} \sqrt{\frac{r_{s}}{r}} \frac{\sin \Theta(r)}{\sqrt{1-\frac{r_{s}}{r}}}, 0\right) \\
& \left(e_{\hat{1}}\right)^{\mu}(\mathbf{x})=\left(e_{\mathrm{r}}\right)^{\mu}(\mathbf{x})=\left(-\sqrt{\frac{r_{\mathrm{s}}}{r}} \frac{\cos \tilde{\Theta}(r)}{\left(1-\frac{r_{s}}{r}\right)}, \cos \Theta(r) \cos \tilde{\Theta}(r)\right. \\
& \left.-\sin \Theta(r) \sin \tilde{\Theta}(r) \sqrt{1-\frac{r_{s}}{r}}, \frac{1}{r} \frac{\sin \Theta(r) \cos \tilde{\Theta}(r)}{\sqrt{1-\frac{r_{s}}{r}}}+\frac{1}{r} \cos \Theta(r) \sin \tilde{\Theta}(r), 0\right) \\
& \left(e_{\hat{2}}\right)^{\mu}(\mathbf{x})=\left(e_{\theta}\right)^{\mu}(\mathbf{x})=\left(\sqrt{\frac{r_{s}}{r}} \frac{\sin \tilde{\Theta}(r)}{\left(1-\frac{I_{s}}{r}\right)}, \cos \Theta(r) \frac{\sin \tilde{\Theta}(r)}{\left(1-\frac{r_{s}}{r}\right)}\right. \\
& \left.-\sin \Theta(r) \cos \tilde{\Theta}(r) \sqrt{1-\frac{r_{s}}{r}}, \frac{1}{r} \cos \Theta(r) \cos \tilde{\Theta}(r)-\frac{1}{r} \frac{\sin \Theta(r) \sin \tilde{\Theta}(r)}{\sqrt{1-\frac{r_{s}}{r}}}, 0\right) \\
& \left(e_{\hat{3}}\right)^{\mu}(\mathbf{x})=\left(e_{\varphi}\right)^{\mu}(x)=(0,0,0,1 / r),
\end{aligned}
$$

where $\cos \Theta(r)=\sqrt{\left(1-\frac{R_{\text {obs }}}{r_{\mathrm{s}}}\left(1-\frac{r_{\mathrm{s}}}{r}\right)\right)}, \sin \Theta(r)=-\sqrt{\frac{\text { obs }_{\text {obs }}}{r_{\mathrm{s}}}\left(1-\frac{r_{\mathrm{s}}}{r}\right)}$. We set conserved energy, $\varepsilon_{\text {photon, }}$ of a photon to its frequency to satisfy equivalence principle, and set the energy per unit mass, $\varepsilon_{\mathrm{obs}}$, of an observer to one, in the units where $\hbar=G=c=1$ since $\varepsilon_{\mathrm{obs}}=\left(1-r_{\mathrm{s}} / r\right) \mathrm{dt} / \mathrm{d} \tau \approx 1$. We set the angular momentum per unit mass $l_{\text {obs }}$ of the observers on a spiraling trajectory as $0.4 \sqrt{r_{\mathrm{s}} r_{\text {Earth }}}$ to put our system in the first-order approximation limit, where $r_{\text {Earth }} \approx 6.4 \times 10^{8} \mathrm{~cm}$ is the radius of Earth, and $r_{s} \approx 1 \mathrm{~cm}$ is its Schwarzschild radius. Besides, we set the (non-radial) launching angle of the photon as $45^{\circ}$ (Fig. 2b) by setting an angular momentum of a photon $I_{\mathrm{ph}}$ as $\frac{\omega r_{\text {Earth }}}{\sqrt{2}}$ so that the radial and polar components of the wave vector have the same value on the surface of Earth, $k^{r}\left(r_{\text {Earth }}\right)=r k^{\varphi}\left(r_{\text {Earth }}\right)$. Here, $\omega$ is an angular frequency of a photon. Details of derivation are provided in the Supplementary Information.

\section{The Wigner rotation under the influence of the gravitational fields}

Upon observation of Eqs. (9) and (10), it is noted that if every parameter is real, then the result of this equation is always real. In other words, WRA $\psi\left(\Lambda, n^{\hat{i}}\right)$ must be $2 m \pi$, where $m$ is an integer. Accordingly, the first and second observer trajectories considered (stationary, radially free-falling) have zero WRAs ${ }^{43}$ since all the parameters are real. In the opposite case where every parameter is not real, the non-zero IWRA for a photon helicity state can be obtained such that

$$
\begin{aligned}
\tilde{\psi}_{\mathrm{inf}} & =2 \operatorname{lm}(\tilde{a})+\left[\frac{2 n^{\hat{1}}}{1+n^{\hat{3}}} \operatorname{Im}(\tilde{\beta})+\frac{2 n^{\hat{2}}}{1+n^{\hat{3}}} \operatorname{Re}(\tilde{\beta})\right] \\
& \equiv \tilde{\psi}_{\mathrm{inf}}^{\text {classical }}+\tilde{\psi}_{\mathrm{inf}}^{\text {quantum }},
\end{aligned}
$$

which corresponds to the last two cases considered, namely freefalling observers with angular momentum in a circular, or in a spiraling trajectory. Here, $\tilde{a}$ and $\tilde{\beta}$ are defined as Supplementary Eq. (34). We note that IWRA consists of a classical geodetic precession around the third axis, $\tilde{\psi}_{\text {inf }}^{\text {classical }}=2 \operatorname{lm}(\tilde{a})$, and a residual quantum phase (or 'residual gauge transformation ${ }^{60 \prime}$ ) induced with the gauge-fixing for a finite irreducible unitary representation, $\tilde{\psi}_{\text {inf }}^{\text {quantum }}=\frac{2 n^{\hat{1}}}{1+n^{\hat{3}}} \operatorname{Im}(\tilde{\beta})+\frac{2 n^{\hat{2}}}{1+n^{\hat{3}}} \operatorname{Re}(\tilde{\beta})$; the WRA for massless particles arises from a consideration of the unitary representation of the transformation of quantum single-particle states under classical Lorentz transformations, and hence manifests itself as a phase factor ${ }^{36,37,43,56}$ depending only on the direction of the photon's momentum but not its frequency. Others in the literature have ascribed the WRA to a residual gauge transformation, and the classical and quantum nature of this non-trivial phase factor remains an open question ${ }^{60}$.

The classical geodetic contribution corresponds to the rotation around the wave vector in the standard frame, where polarization vectors are measured and compared. For the circular-orbit case, parallel transport compensates the rotation induced by spherical coordinates such that spacelike components of the tetrads are rotated by $\theta$ when an observer moves by $-r \theta$, leading to a small (almost zero, see Table 1), total classical geodetic WRA. The classical geodetic effects calculated with tetrads are compared with experimental data reported by Everitt et al. ${ }^{26}$. For the case of a spiraling-orbit, parallel-transport rotates the tetrads around the local third-axis by the angle $2 \Theta^{\text {tetrads }}(r)$, which is defined as

$\sin \Theta^{\text {tetrads }}(r) \approx-\frac{l_{\text {obs }}}{\sqrt{r r_{s}}} \sqrt{1-\frac{r_{s}}{r}}$

where $I_{\text {obs }}$ represents angular momentum per unit mass of an observer. In Fig. 3, we show a conceptual picture to interpret the classical geodetic contribution to IWRA for the case of a spiraling orbit. Since the leading term of classical geodetic precession depends 
Table 1. Comparison of total integrated Wigner rotation angle (WRA) $\psi_{\text {total }}$, classical geodetic contribution to the WRA $\psi_{\text {total }}^{\text {classal }}$, and residual quantum-phase contribution to the WRA $\tilde{\psi}_{\text {total }}^{\text {quantum }}$ (in deg), for a satellite at various altitudes for two types of Earth orbits: (top) circular, (bottom) spiraling.

\begin{tabular}{|c|c|c|c|}
\hline Altitude & $\begin{array}{l}\text { Wigner rotation } \\
\text { angle (WRA) } \\
\text { (classical } \\
\text { geodetic }+ \\
\text { residual } \\
\text { quantum- } \\
\text { phase) } \psi_{\text {total }}\end{array}$ & $\begin{array}{l}\text { Classical } \\
\text { geodetic part } \\
\text { of the WRA } \\
\psi_{\text {total }}^{\text {classical }}\end{array}$ & $\begin{array}{l}\text { Residual } \\
\text { quantum- } \\
\text { phase part of } \\
\text { the WRA } \\
\psi_{\text {total }}^{\text {quantum }}\end{array}$ \\
\hline \multicolumn{4}{|l|}{$\begin{array}{l}\text { Observer in a circular } \\
\text { orbit }\end{array}$} \\
\hline 300 km (NEO) & $2.42 \times 10^{-5}$ & $-6.46 \times 10^{-14}$ & $2.42 \times 10^{-5}$ \\
\hline 2000 km (LEO) & $9.64 \times 10^{-5}$ & $-3.03 \times 10^{-13}$ & $9.64 \times 10^{-5}$ \\
\hline $20,000 \mathrm{~km}$ (MEO) & $-8.78 \times 10^{-7}$ & $-7.02 \times 10^{-13}$ & $-8.78 \times 10^{-7}$ \\
\hline $36,000 \mathrm{~km}(\mathrm{GEO})$ & $-9.93 \times 10^{-5}$ & $-7.61 \times 10^{-13}$ & $-9.93 \times 10^{-5}$ \\
\hline $1.6 \times 10^{11} \mathrm{~km}(r=\infty)$ & $-6.25 \times 10^{-4}$ & $-8.02 \times 10^{-13}$ & $-6.25 \times 10^{-4}$ \\
\hline \multicolumn{4}{|c|}{ Observer in a spiraling orbit } \\
\hline $300 \mathrm{~km}$ (NEO) & 1.14 & 1.14 & $5.32 \times 10^{-6}$ \\
\hline 2000 km (LEO) & 6.31 & 6.31 & $2.41 \times 10^{-6}$ \\
\hline 20,000 km (MEO) & 24.48 & 24.48 & $-1.80 \times 10^{-6}$ \\
\hline 36,000 km (GEO) & 29.31 & 29.31 & $-2.39 \times 10^{-4}$ \\
\hline $1.6 \times 10^{11} \mathrm{~km}(r=\infty)$ & 47.15 & 47.15 & $3.54 \times 10^{-4}$ \\
\hline
\end{tabular}

on only the radial component of wave vector (see Supplementary Information), the system can be simplified by neglecting the angular momentum of a photon - the tetrads are rotated around $\hat{e}_{\phi}$ by the gravitational field and become asymptotically identical to the Schwarzschild coordinate as the photon is observed far away from Earth. Accordingly, the infinitesimal and total classical WRA are described by $\tilde{\psi}_{\text {inf }}^{\text {classical }}=2 \Theta^{\text {tetrads }}(\mathbf{x}+\delta \mathbf{x})-2 \Theta^{\text {tetrads }}(\mathbf{x})$ and $\psi_{\text {total }}^{\text {classical }}=2 \Theta^{\text {tetrads }}\left(\mathbf{x}_{\text {satellite }}\right)-2 \Theta^{\text {tetrads }}\left(\mathbf{x}_{\text {earth }}\right)$, respectively, obtained directly from Eq. (16).

In Table 1, we compare the finite (i) total WRA $\psi_{\text {total, }}$, (ii) classical geodetic contribution of the WRA $\psi_{\text {total }}^{\text {classical }}$, and (iii) the residual quantum phase of the WRA $\psi_{\text {total }}^{\text {quant }}$, for (1) circular and (2) spiraling orbits, obtained by integrating the IWRA $\tilde{\psi}_{\text {inf }}$, $\tilde{\psi}_{\text {inf }}^{\text {classical }}=2 \operatorname{lm}(\tilde{a})$, and $\tilde{\psi}_{\text {inf }}^{\text {quantum }}=\frac{2 n^{1}}{1+n^{3}} \operatorname{Im}(\tilde{\beta})+\frac{2 n^{2}}{1+n^{3}} \operatorname{Re}(\tilde{\beta})$, respectively, along the photon orbit, from the surface of Earth to the altitudes of various Earth orbits. Details of the approximation and interpolation methods employed to compute the total WRA from the IWRA, and to verify their validity are described in detail in the Supplementary Information.

Table 1 shows the total $\psi_{\text {total }}$ WRA as well as its classical geodetic $\psi_{\text {total }}^{\text {classical }}$ and residual quantum $\psi_{\text {total }}^{\text {quantum }}$ phases for (top) a circular and (bottom) a spiraling (massive) observer orbit (here envisioned as a satellite. Further details of the photon's and the observer's orbits can be found in the Supplementary Information). For the circular orbit (with the photon launched non-radially from the Earth, Fig. 2c), Table 1 (top) reveals that the classical (general relativistic) geodetic contribution to the total WRA is effectively zero while the quantum phase yields the dominant contribution in the range of $\left[2.42 \times 10^{-5},-6.25 \times 10^{-4}\right]$ degrees for radial distances ranging from near-Earth orbit (NEO, $300 \mathrm{~km}$ ) to infinity. Figure $4 a$ shows the classical geodetic and residual quantum

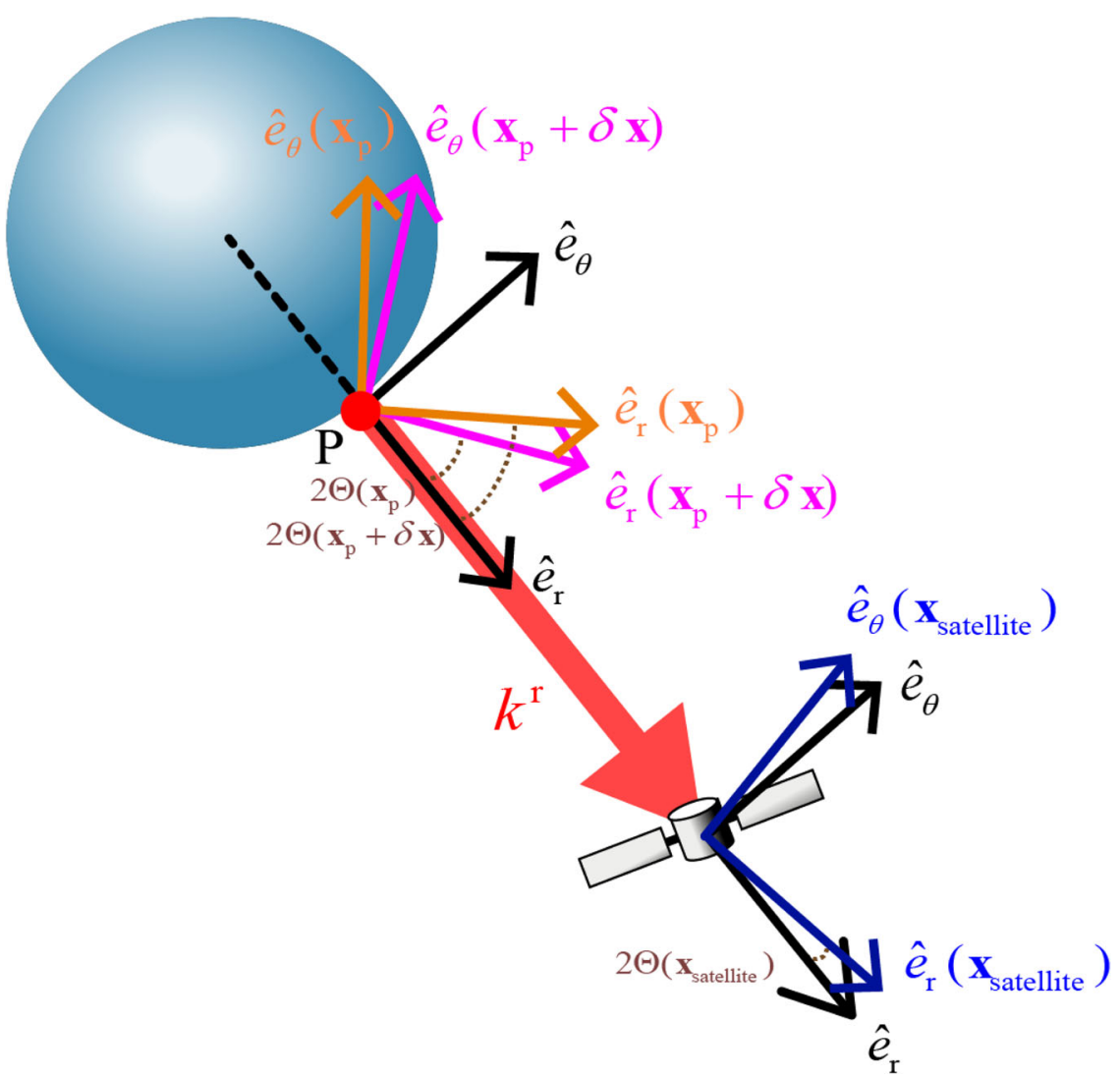

Fig. 3 The conceptual picture of classical geodetic rotation of tetrads. We show the conceptual picture to interpret the tetrads for a free-falling observer with non-zero angular momentum. Since the leading term of classical geodetic IWRA depends on only the radial component of the wave vector, infinitesimal and total classical geodetic Wigner rotations can be seen as $2 \Theta(\mathbf{x}+\delta \mathbf{x})-2 \Theta(\mathbf{x})$ and $2 \Theta\left(\mathbf{x}_{\text {satellite }}\right)-2 \Theta\left(\mathbf{x}_{\text {earth }}\right)$, respectively. 
a

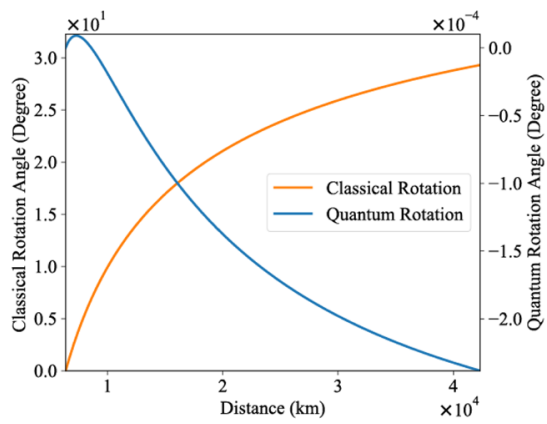

C

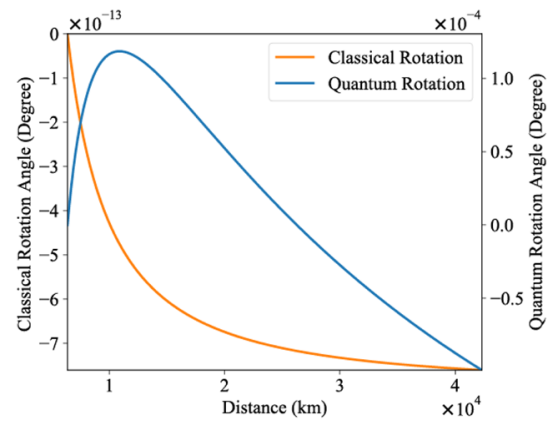

b

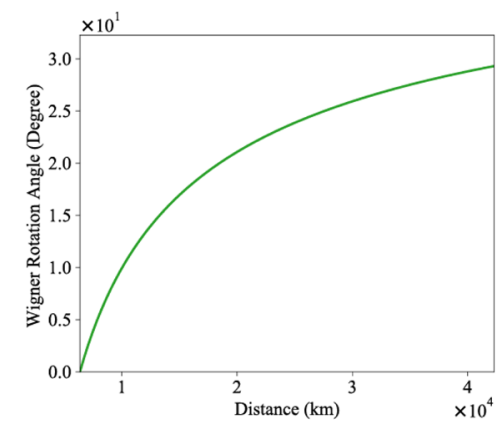

d

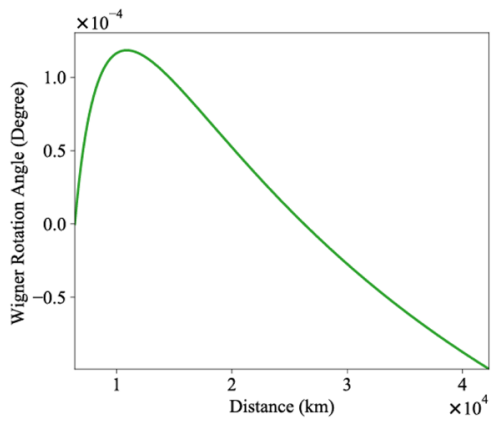

Fig. 4 WRA along distances from Earth. It is shown that the classical geodetic part in the solid orange line, the residual quantum-phase part in the solid blue line, and total WRA in green line for the circular orbit $(\mathbf{a}, \mathbf{b})$ and the spiraling orbit $(\mathbf{c}, \mathbf{d})$.

phase of the total WRA for the circular case. The classical geodetic precession (general relativistic rotation), and the residual quantum phase are represented by the solid orange and solid blue line, respectively. The total WRA is shown in Fig. $4 \mathrm{~b}$.

For the case of the spiraling trajectory, Table 1 (bottom), the opposite is true, namely that the dominant part of the total WRA arises from its classical geodetic contribution. It is confirmed numerically that the total classical WRA $\psi_{\text {total }}^{\text {classical }}$ obtained from two different methods, i.e., by integrating $\tilde{\psi}_{\text {inf }}^{\text {classical }}=2 \operatorname{lm}(\tilde{a})$ along the photon's orbit and directly by using $\psi_{\text {total }}^{\text {classical }}=2 \Theta^{\text {tetrads }}\left(\mathbf{x}_{\text {satellite }}\right)-2 \Theta^{\text {tetrads }}\left(\mathbf{x}_{\text {earth }}\right), \quad$ yield identical numerical values. In addition, there exists a non-zero quantum phase (column four) on the same order of magnitude as in the previous circular case, in the range of $\left[5.32 \times 10^{-6},-3.54 \times 10^{-4}\right]$ degrees for radial distances ranging again from NEO to infinity. The classical geodetic and residual quantum-phase contributions to the total WRA are shown in Fig. $4 c$ and $d$ shows the total WRA.

It is also found that the residual quantum phases have path dependence unlike the classical geodetic cases: for radially emitted photon, while the classical geodetic part of WRA does not change compared to photons with angular momentum, the residual quantum-phase WRA has different values along radial distances ranging again from NEO to infinity in the range of $\left[-3.44 \times 10^{-5},-1.51 \times 10^{-3}\right]$ and $\left[-2.04 \times 10^{-5},-8.55 \times 10^{-4}\right]$ degrees for circular orbits and spiraling orbits, respectively (See Supplementary Table 2).

For standard BB84 protocol, when a horizontal $\left(\hat{e}_{\hat{1}}\right)$ polarization is expected to be detected in a receiver's standard frame, and QBER induced by other factors but WRA are compensated, the total WRA corresponds to an additional QBER of $1.21 \%$ in the case of the LEO and $17.2 \%$ in the case of MEO defined by $\mathrm{QBER}^{\text {classical+quantum }}=\sin ^{2}\left(\psi_{\text {total }}^{\text {classical }}+\psi_{\text {total }}^{\text {quantum }}\right)^{61}$. For the circular trajectory, $\quad \psi_{\text {total }}^{\text {classical }} \approx 0$ and therefore $\mathrm{QBER}^{\text {classical+quantum }} \approx$ $\left(\psi_{\text {total }}^{\text {quantum }}\right)^{2} \sim O\left(\left[10^{-11}, 10^{-7}\right]\right)$ from NEO to infinite radii.
On the other hand, for the spiraling trajectory $\mathrm{QBER}^{\text {classical }+ \text { quantum }}=\sin ^{2}\left(\psi_{\text {total }}\right) \approx \mathrm{QBER}^{\text {classical }}(1+\varepsilon)$, which contains both the dominant classical contribution $\mathrm{QBER}^{\text {classical }}=$ $\sin ^{2}\left(\psi_{\text {total }}^{\text {classical }}\right)$ and a fractional change $\varepsilon$ due to the quantum contribution to the WRA given to lowest order by $\varepsilon \approx$ $2 \psi_{\text {total }}^{\text {quantum }} \sqrt{1-\left(\text { QBER }^{\text {classical }}\right)^{2} / \text { QBER }^{\text {classical }}}$ (see Supplementary Note 10). Inspection of Table 2 shows that $\varepsilon \approx 0.05-0.08 \%$ for almost all radii, including NEO. While for practical applications, this amounts to a small, though the finite contribution to the total QBER, it does serve to illustrate the fundamental limitations to quantum communication arising from the gravitational field, which cannot be avoided in free-falling frames. This result is also consistent with recent analysis, showing that a near-Earth-tospace QKD system that relies on entanglement distribution of photon states could have an additional contribution to its QBER as high as $0.7 \%$ because of spacetime curvature, and these effects are observable with current technologies ${ }^{9}$.

\section{DISCUSSION}

In this paper, we study the Wigner rotation of a photon state in Schwarzschild spacetime to study a rotation of its polarization, considering only the monopole of Earth. While the quadrupole of Earth could induce non-negligible effect on WRA ${ }^{62}$ and ionosphere and Van Allen belt could rotate the polarization vector of a photon, we focus on showing that there is an additional and measurable WRA in addition to the geodetic precession. We calculate the wave vector of the photon to obtain infinitesimal local Lorentz transformations for the four cases of a stationary observer, a free-falling observer with zero angular momentum, and free-falling observers with angular momentum in a circular and spiraling orbit. For the first two cases, the calculated WRA are zero. We calculate the non-zero WRA for the last two cases in 
Table 2. Total QBER and the fractional change $\varepsilon$ induced by quantum WRA for the spiraling Earth Orbit: QBER $^{\text {classical }}=\sin ^{2}\left(\psi_{\text {total }}^{\text {classical }}\right)$, $\mathrm{QBER}^{\text {classical+quantum }}=\sin ^{2}\left(\psi_{\text {total }}\right) \approx \mathrm{QBER}^{\text {classical }}(1+\varepsilon)$,.

\begin{tabular}{lll}
\hline Altitude & QBER $^{\text {classical }}$ & $\varepsilon \approx 2 \psi_{\text {total }}^{\text {quantum }} \sqrt{\left(1-\left(Q^{2}\right.\right.}$ \\
\hline $300 \mathrm{~km}(\mathrm{NEO})$ & $3.92 \times 10^{-4}$ & $5.4 \times 10^{-4}$ \\
$2000 \mathrm{~km}(\mathrm{LEO})$ & $1.21 \times 10^{-2}$ & $4.4 \times 10^{-5}$ \\
$20,000 \mathrm{~km}(\mathrm{MEO})$ & $1.72 \times 10^{-1}$ & $7.9 \times 10^{-4}$ \\
$36,000 \mathrm{~km}(\mathrm{GEO})$ & $2.34 \times 10^{-1}$ & $8.5 \times 10^{-4}$ \\
$1.6 \times 10^{11} \mathrm{~km}(r=\infty)$ & $5.37 \times 10^{-1}$ & $6.6 \times 10^{-4}$ \\
\hline
\end{tabular}

two different ways: (i) by using physically meaningful approximations and (ii) by an interpolation method (see SI) for verification of our results since the differential equation for the photon's trajectory is challenging to solve analytically, and the tetrads for spiraling orbits have a complex a form that inhibits easy physical interpretations. It is found that two different approaches give the same results up to seven significant figures. The circular orbit results in a WRA whose classical geodetic contribution is effectively zero for all practical measurements, yet whose contribution is $2.42 \times 10^{-5}$ degrees at NEO, and $-6.25 \times$ $10^{-4}$ degrees at infinity. Their effect (sine squared value) on the QBER is effectively zero. For the spiraling trajectory, the total WRA is dominated by its large classical geodetic contribution, while the smaller residual quantum phase (or 'residual gauge transformation') is $5.32 \times 10^{-6}$ degrees at NEO and $-3.54 \times 10^{-4}$ degrees at infinity. These results are significantly larger than previous classical geodetic estimations. Furthermore, for the spiraling orbit, the total WRAs (arising predominantly from the classical, geodetic contribution) have angles of 1.13504 degrees at NEO and 47.1469 degrees at infinity and are expected to contribute $1.21 \%$ and $17.2 \%$ to the QBER in the case of LEO and MEO, respectively. In addition, the residual quantum-phase contributions to the QBER for the spiraling orbit constitute a fractional change on the order of $0.05-0.08 \%$ for almost all radii, from NEO to infinity.

It is also interesting to compare these results with the works by Connors et al. ${ }^{63}$, who estimated the polarization rotation angle of $82^{\circ}$ at infinity from the X-rays near a black hole in Cygnus X-1 by using the general relativistic calculations. Our approach can also be applied to the astronomical measurement of the rotation of the photon polarization from the black holes such as recently observed $M 87^{64,65}$. In addition, if one is able to measure the polarization rotation of a photon passing both far from (predominantly classical geodetic contribution to the WRA) and close by (classical geodetic + residual quantum phase) a massive gravitating object, and then take the difference between the two rotation angles, one would have a measure of the residual quantum-phase contribution to the WRA.

\section{METHODS}

\section{Numerical calculation}

Using a Mathematica notebook (Wolfram Research Europe Ltd, Long Hanborough, UK), all of the numerical results in the main text are obtained.

\section{Interpolation method}

We verify the approximations used above by introducing the interpolation method and calculating WRA rotation with the exact tetrad satisfying Supplementary Eq. (64). The Supplementary Eqs. (14) and (64) can be

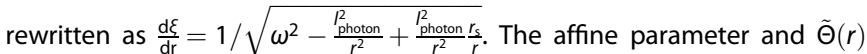
can be written in terms of the distance $r$. By integrating Supplementary Eqs. (64) and (75) from the radius of Earth to the altitudes of orbits with $100 \mathrm{~km}$ intervals and applying interpolation methods, $r(\xi)$ and $\tilde{\Theta}(r)$ are obtained. It is shown that approximation and interpolation methods give the same result up to seven significant figures for the spiraling case.

\section{DATA AVAILABILITY}

The data that support the plots in this paper and other findings of this study are available from the corresponding author upon reasonable request.

\section{CODE AVAILABILITY}

The computer codes and mathematical algorithms that support the plots in this paper and other findings of this study are available from the corresponding author upon reasonable request.

Received: 14 December 2020; Accepted: 7 August 2021; Published online: 18 November 2021

\section{REFERENCES}

1. Yin, J. et al. Entanglement-based secure quantum cryptography over 1,120 kilometres. Nature 582, 501-505 (2020).

2. Yin, J. et al. Satellite-based entanglement distribution over 1200 kilometers. Science 356, 1140 (2017).

3. Ren, J.-G. et al. Ground-to-satellite quantum teleportation. Nature 549, 70-73 (2017).

4. Liao, S.-K. et al. Satellite-to-ground quantum key distribution. Nature 549, 43-47 (2017).

5. Liao, S.-K. et al. Satellite-relayed intercontinental quantum network. Phys. Rev. Lett. 120, 030501 (2018).

6. Armengol, J. M. P. et al. Quantum communications at ESA: towards a space experiment on the ISS. Acta Astronaut. 63, 165-178 (2008)

7. Ursin, R. et al. Space-quest, experiments with quantum entanglement in space. Europhys. N. 40, 26-29 (2009).

8. Rideout, D. et al. Fundamental quantum optics experiments conceivable with satellites-reaching relativistic distances and velocities. Class. Quantum Gravity 29, 224011 (2012).

9. Bruschi, D. E., Ralph, T. C., Fuentes, I., Jennewein, T. \& Razavi, M. Spacetime effects on satellite-based quantum communications. Phys. Rev. D 90, 045041 (2014).

10. Buttler, W. T. et al. Free-space quantum-key distribution. Phys. Rev. A 57, 2379-2382 (1998).

11. Gibson, G. et al. Free-space information transfer using light beams carrying orbital angular momentum. Opt. Express 12, 5448-5456 (2004)

12. Schmitt-Manderbach, T. et al. Experimental demonstration of free-space decoy-state quantum key distribution over 144 km. Phys. Rev. Lett. 98, 010504 (2007)

13. Aolita, L. \& Walborn, S. P. Quantum communication without alignment using multiple-qubit single-photon states. Phys. Rev. Lett. 98, 100501 (2007).

14. Laing, A., Scarani, V., Rarity, J. G. \& O'Brien, J. L. Reference-frame-independent quantum key distribution. Phys. Rev. A 82, 012304 (2010).

15. Zhang, P. et al. Reference-frame-independent quantum-key-distribution server with a telecom tether for an on-chip client. Phys. Rev. Lett. 112, 130501 (2014).

16. Erven, C. et al. Studying free-space transmission statistics and improving freespace quantum key distribution in the turbulent atmosphere. N. J. Phys. 14, 123018 (2012).

17. Vallone, G. et al. Free-space quantum key distribution by rotation-invariant twisted photons. Phys. Rev. Lett. 113, 060503 (2014). 
18. Yin, J. et al. Satellite-based entanglement distribution over 1200 kilometers. Science 356, 1140-1144 (2017).

19. Liao, S.-K. et al. Satellite-to-ground quantum key distribution. Nature 549, 43-47 (2017).

20. Ren, J.-G. et al. Ground-to-satellite quantum teleportation. Nature 549, 70 (2017).

21. Ahn, D. Unruh effect as a noisy quantum channel. Phys. Rev. A 98, 022308 (2018).

22. Ahn, D. Black hole state evolution, final state and Hawking radiation. Class. Quantum Gravity 29, 224007 (2012).

23. Takenaka, $\mathrm{H}$. et al. Satellite-to-ground quantum-limited communication using a 50-kg-class microsatellite. Nat. Photonics 11, 502-508 (2017).

24. de Sitter, W. On Einstein's theory of gravitation and its astronomical consequences. Second Paper. Mon. Not. R. Astron. Soc. 78, 3-28 (1917).

25. Schiff, L. I. Possible new experimental test of general relativity theory. Phys. Rev. Lett. 4, 215-217 (1960).

26. Everitt, C. W. F. et al. Gravity probe B: final results of a space experiment to test general relativity. Phys. Rev. Lett. 106, 221101 (2011).

27. Lense, J. \& Thirring, H. Über den Einfluss der Eigenrotation der Zentralkörper auf die Bewegung der Planeten und Monde nach der Einsteinschen Gravitationstheorie. Phy. Z. 19, 156-163 (1918).

28. Fadeev, P. et al. Gravity probe spin: prospects for measuring general-relativistic precession of intrinsic spin using a ferromagnetic gyroscope. Phys. Rev. D 103, 044056 (2020).

29. Brodutch, A. \& Terno, D. R. Polarization rotation, reference frames, and Mach's principle. Phys. Rev. D 84, 121501 (2011).

30. Brodutch, A., Demarie, T. F. \& Terno, D. R. Photon polarization and geometric phase in general relativity. Phys. Rev. D 84, 104043 (2011).

31. Brodutch, A., Gilchrist, A., Guff, T., Smith, A. R. H. \& Terno, D. R. Post-Newtonian gravitational effects in optical interferometry. Phys. Rev. D 91, 064041 (2015).

32. Fayos, F. \& Llosa, J. Gravitational effects on the polarization plane. Gen. Relativ. Gravit. 14, 865-877 (1982).

33. Zych, M., Costa, F., Pikovski, I. \& Brukner, Č. Quantum interferometric visibility as a witness of general relativistic proper time. Nat. Commun. 2, 505 (2011).

34. Zych, M., Costa, F., Pikovski, I., Ralph, T. C. \& Brukner, Č. General relativistic effects in quantum interference of photons. Class. Quantum Gravity 29, 224010 (2012).

35. Ohnuki, Y. Unitary Representations of the Poincar Group and Relativistic Wave Equations. (World Scientific, 1988).

36. Wigner, E. On unitary representations of the inhomogeneous Lorentz group. Ann. Math. 149-204 (1939).

37. Weinberg, S. The quantum theory of fields. Vol. 1 (Cambridge university press, 1995).

38. Ahn, D., Lee, H.-j, Moon, Y. H. \& Hwang, S. W. Relativistic entanglement and Bell's inequality. Phys. Rev. A 67, 012103 (2003).

39. Terashima, H. \& Ueda, M. Einstein-Podolsky-Rosen correlation in a gravitational field. Phys. Rev. A 69, 032113 (2004).

40. Nakahara, M. Geometry, Topology and Physics. (CRC Press, 2003).

41. Ahn, D. Wigner rotation of a spin $1 / 2$ particle near the event horizon of a schwarzschild black hole. J. Korean Phys. Soc. 51, 470-474 (2007).

42. Alsing, P. M., Stephenson Jr, G. J. \& Kilian, P. Spin-induced non-geodesic motion, gyroscopic precession, Wigner rotation and EPR correlations of massive spin $1 / 2$ particles in a gravitational field. Preprint at https://arxiv.org/abs/0902.1396v1 (2009).

43. Alsing, P. M. \& Stephenson Jr, G. J. The Wigner rotation for photons in an arbitrary gravitational field. Preprint at https://arxiv.org/abs/0902.1399 (2009).

44. Weinberg, S. Gravitation and Cosmology: Principles and Applications of the General Theory of Relativity. (John Wiley \& Sons, Inc., 1972).

45. De Felice, F. \& Clarke, C. J. S. Relativity on Curved Manifolds. (Cambridge University Press, 1992).

46. Schwinger, J. Energy and momentum density in field theory. Phys. Rev. 130, 800 (1963).

47. Birrell, N. D. \& Davies, P. C. W. Quantum Fields in Curved Space. (Cambridge University Press, 1982).

48. Alsing, P. M. \& Milburn, G. J. Teleportation with a uniformly accelerated partner. Phys. Rev. lett. 91, 180404 (2003).

49. Fuentes-Schuller, I. \& Mann, R. B. Alice falls into a black hole: entanglement in noninertial frames. Phys. Rev. Lett. 95, 120404 (2005).

50. Alsing, P. M., Fuentes-Schuller, I., Mann, R. B. \& Tessier, T. E. Entanglement of Dirac fields in noninertial frames. Phys. Rev. A 74, 032326 (2006).

51. Alsing, P. M. \& Fuentes, I. Observer-dependent entanglement. Class. Quantum Gravity 29, 224001 (2012).

52. Hawking, S. W. Particle creation by black holes. Comm. Math. Phys. 43, 199-220 (1975).

53. Unruh, W. G. Notes on black-hole evaporation. Phys. Rev. D. 14, 870 (1976).
54. Hartle, J. B. Gravity: An Introduction to Einstein's General Relativity (American Association of Physics Teachers, 2003).

55. Misner, C. W., Thorne, K. S. \& Wheeler, J. A. Gravitation. (Macmillan, 1973).

56. Caban, P. \& Rembieliński, J. Photon polarization and Wigner's little group. Phys. Rev. A 68, 042107 (2003).

57. Alsing, P. M. \& Milburn, G. J. Lorentz invariance of entanglement. Q. Info. Comm. 2, 487-512 (2002).

58. Han, D., Kim, Y. S. \& Son, D. Unitary transformations of photon polarization vectors. Phys. Rev. D 31, 328-330 (1985).

59. Gingrich, R. M., Bergou, A. J. \& Adami, C. Entangled light in moving frames. Phys. Rev. A 68, 042102 (2003).

60. Kohlrus, J., Louko, J., Fuentes, I. \& Bruschi, D. E. Wigner phase of photonic helicity states in the spacetime of the Earth. Preprint at https://arxiv.org/abs/1810.10502 (2019).

61. Muga, N. J., Ferreira, M. F. S. \& Pinto, A. N. QBER estimation in QKD systems with polarization encoding. J. Light. Technol. 29, 355-361 (2010).

62. Roh, K.-M., Kopeikin, S. M. \& Cho, J.-H. Numerical simulation of the postNewtonian equations of motion for the near Earth satellite with an application to the LARES satellite. Adv. Space Res. 58, 2255-2268 (2016).

63. Connors, P. A., Piran, T. \& Stark, R. F. Polarization features of X-ray radiation emitted near black holes. Astrophys. J. 235, 224-244 (1980).

64. Akiyama, K. et al. First M87 event horizon telescope results. IV. Imaging the central supermassive black hole. Astrophys. J. Lett. 875, L4 (2019).

65. Collaboration, E. H. T. et al. Gravitational test beyond the first post-Newtonian order with the shadow of the M87 black hole. Phys. Rev. Lett. 125, 141104 (2020).

\section{ACKNOWLEDGEMENTS}

This work was supported by Institute for Information \& communications Technology Promotion(IITP) grant funded by iC2S2 grants from the Korean Ministry of Science and Future Planning (MSIP) IITP 2017-0-00266 and the US Air Force Asian Office of Aerospace Research \& Development (AFOSR/AOARD) FA2386-17-1-4070, and Institute for Information \& communications Technology Promotion(IITP) grant (IITP-2018-20150-00385 ITRC Center for Quantum Communications). D.A. was also supported by Korea National Research Foundation (NRF) grant No. NRF-2020M3E4A1080031: Quantum circuit optimization for efficient quantum computing, NRF grant No. NRF2020M3H3A1105796, ICT R\&D program of MSIT/IITP 2021-0-01810 and AFOSR grant FA2386-21-1-0089. WAM was supported in part under the 2018 VFRP Program at AFRL/RI and Griffiss Institute. P.M.A. would like to acknowledge support from the Air Force Office of Scientific Research (AFOSR). Any opinions, findings, and conclusions or recommendations expressed in this material are those of the author(s) and do not necessarily reflect the views of the Air Force Research Laboratory.

\section{AUTHOR CONTRIBUTIONS}

D.A. proposed a theoretical model for a photon state in free-space quantum communication and wrote the main manuscript. H.N. worked out the tetrads, the photon trajectories, numerical simulations, and wrote the main manuscript and Supplementary Information. P.M.A. worked out the development of Wigner rotations for a photon state in curved spacetime. W.M. suggested the basic idea of photon trajectories in curved spacetime and contributed to the discussion of the main manuscript. N.P. contributed to the optical communication protocol. All authors reviewed the manuscript.

\section{COMPETING INTERESTS}

The authors declare no competing interests.

\section{ADDITIONAL INFORMATION}

Supplementary information The online version contains supplementary material available at https://doi.org/10.1038/s41534-021-00471-6.

Correspondence and requests for materials should be addressed to Paul M. Alsing or Doyeol Ahn.

Reprints and permission information is available at http://www.nature.com/ reprints

Publisher's note Springer Nature remains neutral with regard to jurisdictional claims in published maps and institutional affiliations. 
Open Access This article is licensed under a Creative Commons Ac Attribution 4.0 International License, which permits use, sharing,
adaptation, distribution and reproduction in any medium or format, as long as you give appropriate credit to the original author(s) and the source, provide a link to the Creative Commons license, and indicate if changes were made. The images or other third party material in this article are included in the article's Creative Commons license, unless indicated otherwise in a credit line to the material. If material is not included in the article's Creative Commons license and your intended use is not permitted by statutory regulation or exceeds the permitted use, you will need to obtain permission directly from the copyright holder. To view a copy of this license, visit http://creativecommons. org/licenses/by/4.0/.

(c) The Author(s) 2021 